Article

\title{
Broad-Range Hydrogel-Based pH Sensor with Capacitive Readout Manufactured on a Flexible Substrate
}

\author{
Krister Hammarling 1,*(D), Magnus Engholm ${ }^{1}$, Henrik Andersson 1 (D) Mats Sandberg ${ }^{1,2}$ (D) and \\ Hans-Erik Nilsson 1 (iD \\ 1 Department of Electronics, Mid Sweden University, Holmgatan 10, 85170 Sundsvall, Sweden; \\ magnus.engholm@miun.se (M.E.) ; henrik.andersson@miun.se (H.A.); mats.sandberg@ri.se (M.S.); \\ hans-erik.nilsson@miun.se (H.-E.N.) \\ 2 RISE Acreo AB, Bredgatan 33, 60117 Norrköping, Sweden \\ * Correspondence: krister.hammarling@miun.se; Tel.: +46-010-1428717
}

Received: 17 June 2018; Accepted: 23 July 2018; Published: 25 July 2018

\begin{abstract}
Environmental monitoring of land, water and air, is an area receiving greater attention because of human health and safety concerns. Monitoring the type of pollution and concentration levels is vital, so that appropriate contingency plans can be determined. To effectively monitor the environment, there is a need for new sensors and sensor systems that suits these type of measurements. However, the diversity of sensors suitable for low, battery powered- and large area sensor systems are limited. We have manufactured and characterized a flexible $\mathrm{pH}$ sensor using laser processing and blade coating techniques that is able to measure $\mathrm{pH}$ between 2.94 and 11.80 . The sensor consists of an interdigital capacitance with a $\mathrm{pH}$ sensitive hydrogel coating. Thin sensors can reach $95 \%$ of their final value value within $3 \mathrm{~min}$, and are stable after $4 \mathrm{~min}$. Good repeatability was achieved in regard to cycling of the sensor with different $\mathrm{pH}$ and multiple measurements from dry state. We have also studied the relation between an interdigital capacitance penetration depth and hydrogels expansion. We believe that our passive sensor is suitable to be used in low power and large area sensor networks.
\end{abstract}

Keywords: interdigital; hydrogel; penetration depth; $\mathrm{pH}$; sensor; coating; thin film; laser ablation; oligo ( $\beta$-amino esters)

\section{Introduction}

The increase of environmental problems on land, in water and air from pollution, over fertilization and insufficient waste-water treatment are receiving greater attention in the technical and scientific area. Contaminants tend to accumulate in the environment, which eventually becomes a hazard to most living organisms [1]. Therefore, it is vital to determine the type of pollution and the concentration, such that appropriate actions can be made [2,3]. Specific areas of interest for monitoring include advanced industrial processes, aquatic biological communities and waste-stream effluents [4]. Heavy metals can spread from waste-stream effluents in landfills, recycling areas and mine deposits, which contaminate the surrounding environment [5-9]. Connel et al. [10] could show that there is a link between desorption of heavy metals and the $\mathrm{pH}$ level of the surrounding soil and water. Hence, by measuring the $\mathrm{pH}$ level, the level of heavy metal leakage in the environment can be monitored.

$\mathrm{pH}$-levels can be measured by using for example, litmus papers, $\mathrm{pH}$ glass electrodes, $\mathrm{pH}$ sensitive polymers, optical $\mathrm{pH}$ sensors, fluorimetric $\mathrm{pH}$ sensors, radiometric $\mathrm{pH}$ sensors and ion sensitive FET $\mathrm{pH}$ sensors [11]. In the field of environmental monitoring there is a special need for distributed sensors to cover large areas. Printed sensors on flexible substrates can offer cost effective manufacturing of sensors 
for environmental monitoring on a large scale. In addition, printed sensors with a stimuli responsive hydrogel used as a sensing layer can show high sensitivity to small variations of environmental parameters such as $\mathrm{pH}$, temperature, moisture and ions etc. Flexible $\mathrm{pH}$ sensors that use Iridium Oxide, carbon nanotubes/polyaniline as well as hydrogel as sensing layers have previously been reported [12-14]. However, most of the $\mathrm{pH}$-sensors reported so far are not fully passive components, often complex to manufacture and therefore not optimal for low energy systems or mass producing.

One promising class of pH-sensitive hydrogels is acryl terminated oligo beta-amino ester's (AOBAE). This biocompatible AOBAE, which is synthesized from 1,3-butanediol diacrylate (1,3-BDDA) and piperazine (PIP) is easy to prepare and without the need for any solvents [15]. Here we demonstrate a printed interdigital capacitive $\mathrm{pH}$ sensor that utilizes such $\mathrm{pH}$ sensitive AOBAE as a sensing material. The resulting solvent uptake within the AOBAE alters the relative permittivity in/of the surroundings of the metal layer, which is detected as a change in the capacitance. We also investigated the relation between the hydrogels thickness change and the penetration depth of the electric field of the interdigital capacitor. This relation has, to our knowledge, not been addressed previously. The produced sensor is found to be sensitive to $\mathrm{pH}$ levels between 2.94 and 11.80 and with a deviation of less than $\pm 0.7 \%$ between repeated $\mathrm{pH}$ measurements of $\mathrm{pH} 7.12$.

\section{Theory}

The hydrogel will respond to $\mathrm{pH}$ level by uptaking or releasing water, resulting in a swelling/deswelling of the hydrogel and an effective relative permittivity change. The change in permittivity is detected by an interdigital capacitor structure. Capacitive sensors are used in a wide range of sensing applications, for example non-destructive testing (NDT), chemical sensing, bio-sensing [16], humidity and moisture sensing [17], monitoring of curing processes [18], wireless sensing [19] and surface acoustic waves (SAW) [20]. An overview of the most important concepts related to interdigital electrode sensors are available in references [16,21-26].

\subsection{Interdigital Capacitance Sensors with Hydrogels}

In general, stimuli-responsive hydrogels can change their volume significantly in response to small variations of different environmental parameters, such as $\mathrm{pH}$, temp, moisture, current, ions etc. [27-31]. Sensors based on hydrogel will, depending on stimuli ( $\mathrm{pH}$, temperature, magnetism etc.), uptake different amounts of water that results in an effective relative permittivity change. The relative permittivity, $\epsilon_{r}$, of water is 80 and the hydrogel (AOBAE) used in this work has a relative permittivity of 5.5. A change of the permittivity within the capacitors electric field changes the capacitance and therefore it is possible to correlate the stimuli of the hydrogel to a change in capacitance.

The interdigital capacitor consists of two electrodes with interspersed "fingers", with finger length $L$, electrode width $W$ and gap $G$, as shown in Figure 1. The sensor structure consists of the interdigital capacitor, an electrically insulating layer, $D_{P}$ (to avoid short circuit) and the hydrogel sensing layer $D$.

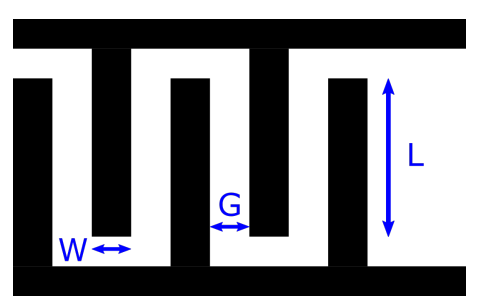

Figure 1. Layout of an interdigital capacitor where $W$ is the width of the electrode, $G$ the gap between two electrodes and $L$ is the length of the electrodes. 
The capacitance is given by Equation (1) [32]

$$
C=\epsilon_{r} \epsilon_{0} \frac{A}{G}
$$

where $\epsilon_{r}$ is the relative permittivity of the medium and $\epsilon_{0}$ is the dielectric constant, $A$ the effective electrode area $(A=L W n / 2$, where $\mathrm{n}$ is the number of electrodes in the capacitance) and $G$ the effective spacing $G$ between the electrodes.

It is important to consider the penetration depth of the electric field out from the electrodes as this will determine how the hydrogel layer and water will affect the capacitance. The electric field penetration depth, $E_{P D}$ is defined as where (at a distance $z$ ) the capacitance $C\left(z=E_{P D}\right)$ equals $3 \%$ of the difference between the highest and the lowest values of the terminal impedance [33], see Figure 2. The distance $E_{P D}$ can be calculated as one third of the width of the electrode plus the gap between electrodes, $E_{P D} \approx(W+G) / 3$ [34].

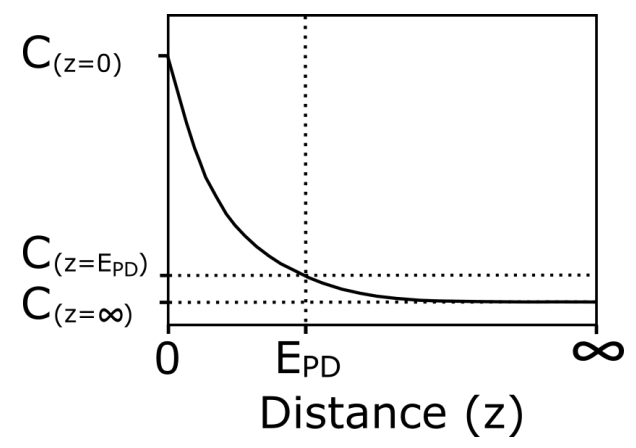

Figure 2. Relation between capacitance and the distance to a material under test, which is placed a distance $z$ from an interdigital capacitance. The distance $z$ is orthogonal to the capacitance $x-y$ plane.

Because the hydrogel thickness change $(\Delta D)$ depends on the stimuli, it is important to consider how the capacitance is affected by this thickness change. Note that the protective layer thickness, $D_{P}$, is neglected in the following discussion of three different cases displayed in Figure 3

(a) If $E_{P D}<D$, the capacitance will be affected only by the permittivity change that occurs in the hydrogel by the uptake or release of water (Figure 3a). The total capacitance will be $C_{t o t}=E_{P D} C_{P H}$, where $C_{P H}$ is defined as the capacitance of the hydrogel per unit thickness.

(b) If $E_{P D}>D+\Delta D$, the total capacitance consists of two parts $C_{1}$ and $C_{2}$. The capacitance contribution from $C_{1}$ is affected by the effective relative permittivity within the hydrogel and $C_{2}$ is affected from the relative permittivity of the surrounding media (water), see Figure $3 \mathrm{~b}$. The total capacitance then becomes $C_{t o t}=C_{1}+C_{2}=(D+\Delta D) C_{P H}+\left[E_{P D}-(D+\Delta D)\right] C_{W}$, where $C_{W}$ is defined as the capacitance of the water per unit thickness.

(c) For the case when $E_{P D}<D+\Delta D_{\max }$ and $E_{P D}>D$, which means that the thickness of the hydrogel is very close to the depth of the electric field, the capacitance will alternate between cases (a) or (b) depending on the thickness change of the hydrogel, see Figure 3c.

Case (a) gives a predictable responsitivity to stimuli change, as $E_{P D}$ is fully contained within the hydrogel layer. The effect of stimuli change will then only come from the effective relative permittivity change within the hydrogel. The change in capacitance is not as easy to predict for cases (b) and (c). This is beacuse the change in capacitance is also affected by the relative permittivity of the second layer (here denoted as water, but can be any other substance). 


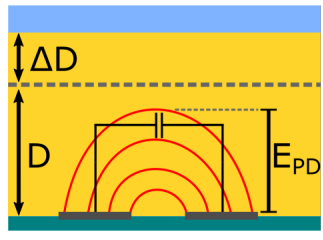

a

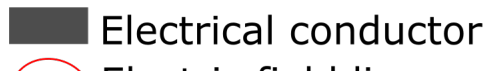
ก Electric field lines $\dashv \vdash$ Electrical equivalent circuit

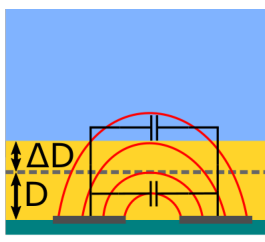

b

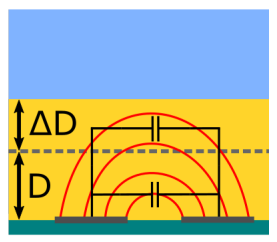

$c=a$ or $b$

\section{Water Hydrogel}

Figure 3. Principle function for interdigital capacitors coated with a hydrogel and their equivalent electric circuit. $D$ represent the minimum thickness of the hydrogel, $\Delta D$ is the thickness change of the hydrogel and $E_{P D}$ is the electric field penetration depth. Case (a) is a single layer sensor, where the sensing hydrogel layer $(D+\Delta D)$ is always thicker then $E_{P D}$; Case $(\mathbf{b})$ is a double layer sensor, i.e., two different dielectrics (hydrogel and water) within the sensing penetration depth $\left(E_{P D}\right)$ contributes to the total capacitance; Case (c) shows a situation where the thickness of the hydrogel layer is close to the penetration depth of the electric field. This result represent a mixed sensor that, depending on circumstances can act as a double layer sensor (hydrogel contracted) and a single layer sensor (hydrogel expanded). For simplicity the electric protection layer $D_{P}$ is omitted from this figure.

\subsection{Simulation}

A numerical simulation have been conducted to visualize the behaviour of the three different sensor cases displayed in Figure 3. The simulation assumes that the electric field strength is equal in the whole penetration depth $E_{P D}$, though in reality the electric field is strongest closest to the electrical conductors and decays exponentially with distance [25]. Also, the insulator layer $D_{P}$ is omitted from this simulation. In the simulation the total sensor capacitance $C_{\text {tot }}$ is calculated as

$$
C_{\text {tot }}=C_{1}+C_{2} \begin{cases}C_{2}=0 & \text { if } D+\Delta D \geqslant E_{P D} \text { (single layer sensor) } \\ C_{2}=C_{2} & \text { if } D+\Delta D<E_{P D} \text { (double layer sensor) }\end{cases}
$$

where $C_{\text {tot }}$ is the total capacitance, $C_{1}$ is the capacitance contribution from the hydrogel layer and $C_{2}$ is capacitance contribution originating from the outside of the hydrogel (e.g., water or air). A complete description of the calculation for the simulation can be found in the Appendix A.

In the simulation shown in Figure 4, we have used three different start thicknesses of the hydrogel; 100/0\% (blue dots, solid line), 60/40\% (green diamonds, short dashed line) and 20/80\% (grey triangles, dashed line). The number (e.g., 100/0) represent the thickness of the first (hydrogel) and second (water) layers in percent of the total electric field penetration depth. For example, in the case of the dual layer sensor (Figure 3 b) we have used $20 / 80 \%$, which means that $20 \%$ of the total electric field penetration depth, $E_{P D}$ is within the hydrogel layer $\left(C_{1}\right)$ and $80 \%$ is within the water layer $\left(C_{2}\right)$. These values are given for a dry sensor. Figure 4 shows how a sensor in the three cases from Figure 3 (single, dual and mixed layer sensor) will behave when the thickness of the hydrogel increase from 1 to $100 \%$.

From the simulation, one can see that for the single layer case (a), where $D \geqslant E_{P D}$ (blue dots, solid line) and the whole electric field is contained within the hydrogel, the capacitance starts at a low value and increases linearly with increasing water uptake in the hydrogel, refer to Figure 3a. For case (b), the double layer sensor where $D+\Delta D<E_{P D}$ (grey triangles, dashed line), the electric field reach outside of the hydrogel layer and is also affected by the surrounding medium (water). The capacitance starts at a high value and decreases linearly with increasing water uptake in the hydrogel, refer to Figure $3 b$. For a mixed layer sensor (green diamonds, short dashed line, refer to Figure $3 c$ the sensor will act as a combination of the sensors in case (a) or (b). This sensor starts as a dual layer sensor $\left(D+\Delta D<E_{P D}\right)$ for low water uptake and at the point where the hydrogel thickness have increased 
such that $D+\Delta D \geqslant E_{P D}$ it will act as a single layer sensor. The slope for the double layer sensor is less then for the single layer sensor. The reason is there are two capacitances, $C_{1}$ and $C_{2}$, adding to the total capacitance though they are working opposite to each other. When capacitance $C_{1}$ increase with hydrogel thickness, capacitance $C_{2}$ will decrease at the same time. $C_{2}$ has a larger capacitance value per unit thickness change compared to $C_{1}$. Due to the ambiguous nature of the mixed layer sensor, where one capacitance value can represent two different $\mathrm{pH}$-levels, this sensor is not very suitable for sensing applications.

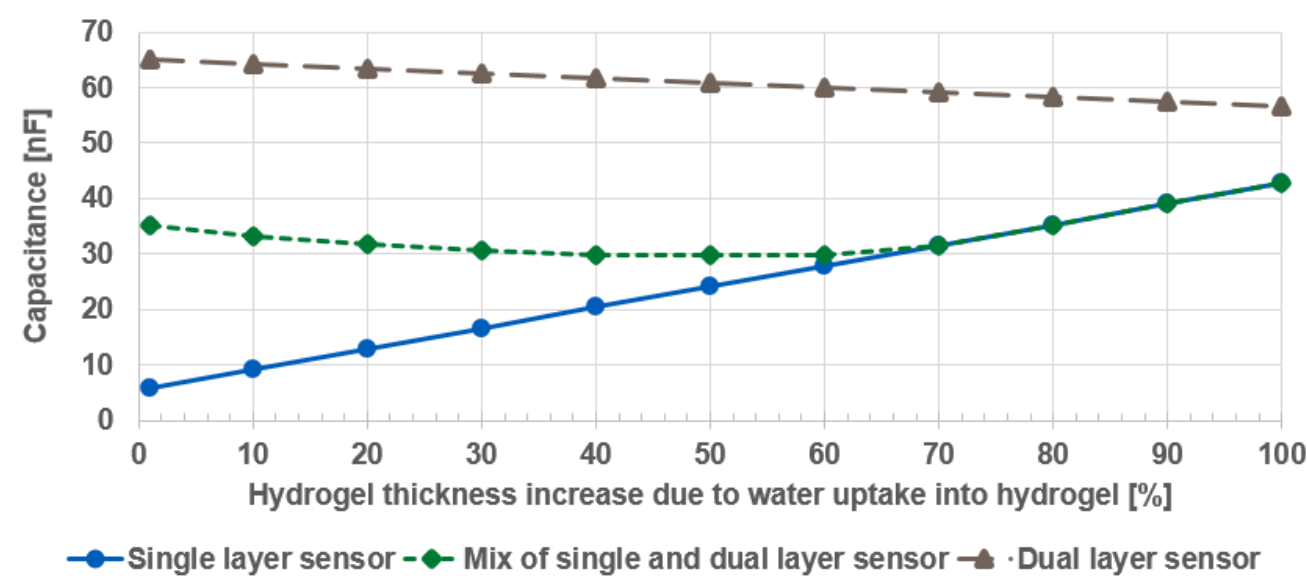

Figure 4. Simulation of the behavior depending of type of sensor, single layer sensor (Figure 3a), double layer sensor (Figure 3b) and a mixed layer sensor (Figure 3c). The simulation calculates the capacitance as a function of the thickness increase from 1 to $100 \%(100 \%=$ twice the thickness). It can be seen that the behaviour of the sensor depends on how the sensor is built, as a single, dual or a mixed layer sensor.

\section{Experimental}

\subsection{Materials}

1,3-butanediol diacrylate (1,3-BDDA), piperazine (PIP), diphenyl(2,4,6-trimethylbenzoyl) phosphine oxide (TPO) and chemicals to make Britton-Robinson buffer (BRB) [35] were purchased from Merck and used without further purification. Gold target used was SC502-314A from Quorum Technologies (Laughton, UK). Mylar film (DuPont Teijin Films, Chester, VA, USA). SU8 2000.5 (Microchem, Westborough, MA, USA).

\section{2. $A O B A E$}

We synthesized an AOBAE with mole ratio of 2:1 (1,3-BDDA:PIP respectively) by dissolving PIP into 1,3-BDDA during stirring at $60{ }^{\circ} \mathrm{C}$, without the use of any solvents. Stirring continued until there were no visible PIP crystals in the solution, and then for another $30 \mathrm{~min}$, making it a total of $120 \mathrm{~min}$. The solution was then left to cool down to room temperature before further processing. The preparation of AOBAE was made analogically with the authors previous work on characterization of $\mathrm{pH}$ sensitive AOBAE [15]. The AOBAE was measured to have a relative permittivity of 5.5 according to the formula $\epsilon_{r}=C / C_{0}$ [36]. The relative permittivity measurement was made in air, with an expected negligible difference compared to vacuum. When the AOBAE is submerged in an acidic liquid, the nitrogen in the peperazine ring gets protonated. As the AOBAE becomes more protonated, the uptake of water will increase and the hydrogel expands.

\subsection{Instruments and Manufacturing of Sensors}

Figure 5 shows the structure of the developed $\mathrm{pH}$-sensor. The sensors were manufactured on a $105 \mu \mathrm{m}$ thick Mylar (PET) film, where a $30 \mathrm{~nm}$ thin gold layer was sputtered on the Mylar substrate by 
using a Quorum Q150T ES sputter (Quorum Technologies, UK). An interdigital capacitance structure was patterned by using a $355 \mathrm{~nm}$ nano-second pulsed laser ablation system allowing for a spatial resolution in the order of $10 \mu \mathrm{m}$. In the present design, the width $(W)$ of the conductive lines and distance $(G)$ between the lines were $120 \mu \mathrm{m}$ and $40 \mu \mathrm{m}$ respectively, see Figure 1 . The interdigital capacitance sensor was designed with a total of 75 lines providing an initial, dry capacitance in the order of $15 \mathrm{pF}$. The performance of the final $\mathrm{pH}$-sensor will depend on the choice of width $W$ and distance $G$ and will be further discussed in Section 5. After laser patterning, a thin layer of SU8 2000.5 was coated on the thin gold layer to act as an electrically insulating layer and prevent direct contact between the $\mathrm{pH}$-solution and the conductive lines. The thickness of the insulating layer was $4 \pm 1 \mu \mathrm{m}$ after soft-baking at $90{ }^{\circ} \mathrm{C}$ for $1 \mathrm{~min}$, UV-curing with a $365 \mathrm{~nm}$ LED for $10 \mathrm{~min}$ and hard baking at $160{ }^{\circ} \mathrm{C}$ for $10 \mathrm{~min}$. The active, $\mathrm{pH}$-sensitive ABOAE layer was deposited by a blade coating technique using a Zehtner ZUA 2000.80 universal applicator (Zhentner, Sissach, Switzerland), while the substrate was held on a vacuum table. Sensors with three different thicknesses of AOBAE were manufactured and evaluated, $5.5 \pm 3 \mu \mathrm{m}, 18.5 \pm 3.5 \mu \mathrm{m}$ and $30.5 \pm 7 \mu \mathrm{m}$. Curing of the SU8 and AOBAE were performed under ambient conditions by using a $365 \mathrm{~nm}$ UV-LED (M365F1, Thorlabs, Newton, MA, USA) with an intensity of $1.9 \mathrm{~mW} / \mathrm{cm}^{2}$.
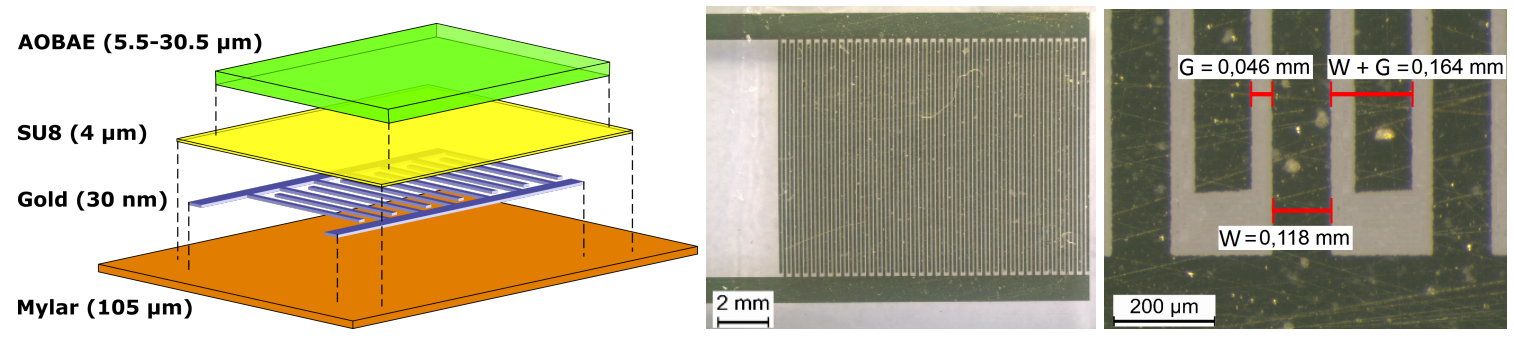

Figure 5. Schematic of the different sensor layers and their thicknesses (left picture). Layers from bottom and up are, Mylar as substrate, interdigital pattern made of gold, SU8 as electric protection layer and AOBAE is the $\mathrm{pH}$ sensitive layer. The middle pictures is a photo of a manufactured sensor (with SU8 and AOBAE on top). The right picture shows an enlargement of the middle pictures lower part, with measurements of conductor width, $W$, and the gap, $G$, between the conductors.

\subsection{Capacitance Measurements}

The interdigital $\mathrm{pH}$-sensors were characterized by submerging each sensor in liquids with defined pH levels. The capacitance values were measured using an HP 4284A RCL meter (Keysight Technologies, Santa Rosa, CA, USA) controlled by a LabView program (National Instruments, Austin, TX, USA). The sensor was held constant in a holder with all cables fixed and kept as short as possible. A motorized labjack was used to lift up/down the different pH-buffers as seen in Figure 6, without disturbing the measurement setup. Before measurement of the sensor capacitance, the cables and holder (without sensor) was measured and values subtracted from all measurement results. For all $\mathrm{pH}$ measurements, $\mathrm{BRB} \mathrm{pH}$ buffer solutions with $\mathrm{pH}$ values ranging between 2.94 and 11.80 were used. Reference $\mathrm{pH}$ measurements were performed on the buffers using a Metrohm $632 \mathrm{pH}$-meter (Metrohm AG, Herisau, Switzerland).

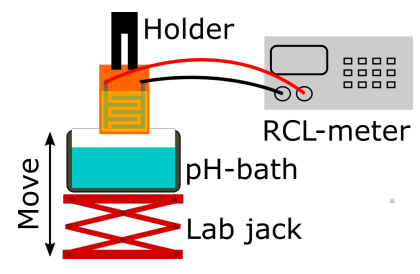

Figure 6. Schematic of the $\mathrm{pH}$-measurement setup. 


\section{Results}

Figure 7 shows the capacitance at a $\mathrm{pH}$ of 7.12 for three sensors with different hydrogel layer thicknesses, 5.5, 18.5 and $30.5 \mu \mathrm{m}$ respectively. In the graph, notice the followings: First, the time to reach a stable capacitance value is longer for a thicker AOBAE layer because the uptake of water is a diffusion controlled process [27,37]; Second, a thinner AOBAE layer results in a higher maximum capacitance. This is because the thinner sensors ( 5.5 and $18.5 \mu \mathrm{m}$ ) also has a capacitance contribution from water; Third, for the two sensors with the thinner AOBAE layers we can see a higher initial rate increase for the capacitance. A distinct knee at $8 \mathrm{~s}$ and $10 \mathrm{~s}$, respectively (marked by arrows in the inset), is observed after which the rate becomes lower, see inset of Figure 7. The stable point of each sensor is marked in the figure with black dots and is defined as where the capacitance deviation stays within $\pm 1 \%$ of the mean value for at least $10 \mathrm{~min}$. The time to reach the stable capacitance value (stabilization time) was approximately 4, 9 and $25 \mathrm{~min}$, for the 5.5, 18.5 and $30.5 \mu \mathrm{m}$ thick films, respectively.

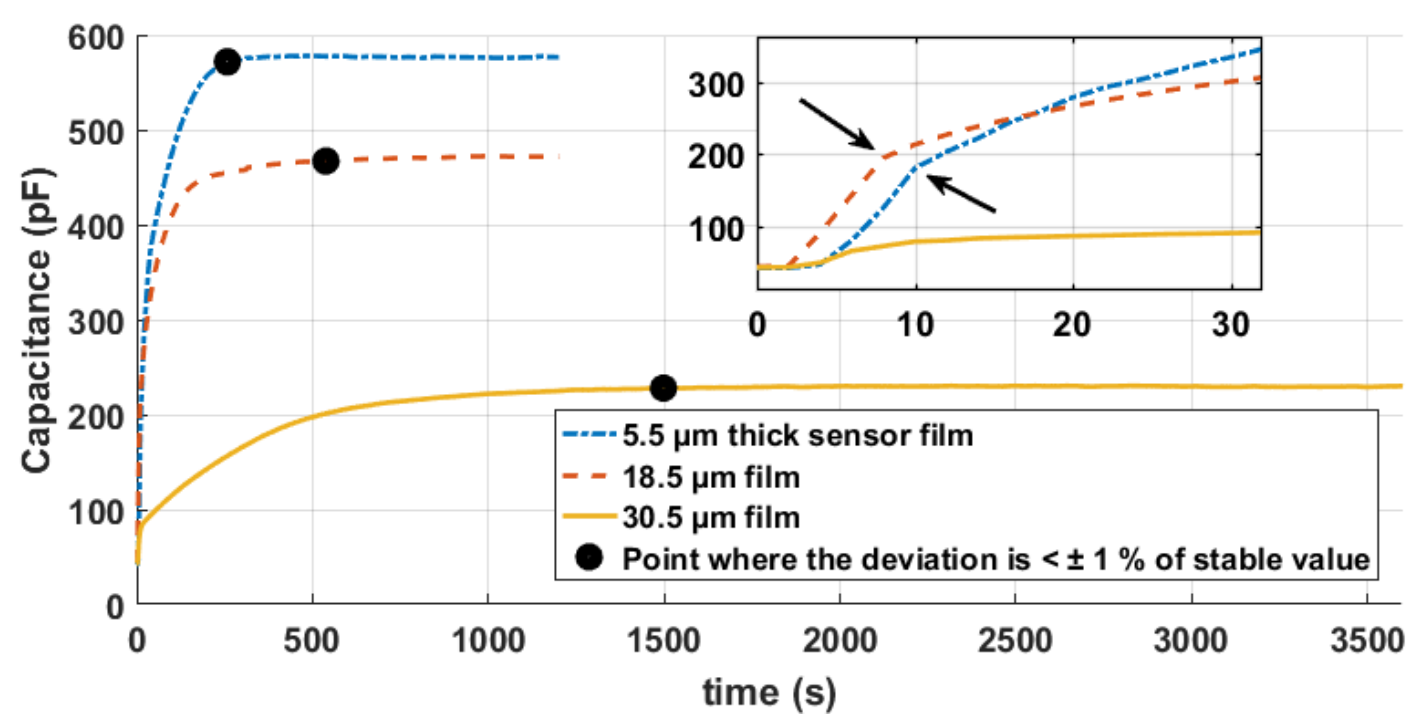

Figure 7. Capacitance response to $\mathrm{pH} 7.12$ for three sensors with different thicknesses of the sensing AOBAE layer 5.5, 18.5 and $30.5 \mu \mathrm{m}$. Thinner sensors have a higher capacitance and reach stable value faster then thicker sensors. This, because the thinner sensors (5.5 and $18.5 \mu \mathrm{m}$ ) also measure upon water directly and water absorption is a diffusing process. The inset show the rise time for the three sensors, where the the time before the arrows (at 8 and $10 \mathrm{~s}$ ) are when the sensors are in the immersion phase (i.e., not fully covered by the $\mathrm{pH}$ solution). Black dots are points where the capacitance of each sensor is considered stable.

Figure 8 shows measurements on a sensor with a sensing layer thickness of $18.5 \mu \mathrm{m}$. The sensor has been measured 5 times in $\mathrm{pH} 7.12$ and 5 times in $\mathrm{pH} 8.38$, in the order 7.12, 8.38, 7.12, .. Between every test the sensor was completely dried (dried until the capacitance value returned to the first, dry value before test, approximately $41 \mathrm{pF}$ ). The error bars show the standard deviation at four different times, 300, 600 , 900 and $1200 \mathrm{~s}$. The standard deviations at $1200 \mathrm{~s}$ are 3.37 and $4.69 \mathrm{pF}$ for $\mathrm{pH} 7.12$ and 8.38 , respectively. This corresponds to a deviation of the final value that is less then $\pm 0.7 \%$ and $\pm 1.1 \%$ respectively.

Figure 9 shows a comparison between a single layer sensor (refer to Figure 3a) and a double layer sensor (refer to Figure 3b) with sensor layer thicknesses of 30.5 and $5.5 \mu \mathrm{m}$ respectively. The sensors were kept in the given solution for $30 \mathrm{~min}$ in order to reach the stable point of the thicker sensor. The deviation between the three cycles is \pm 0.5 and $\pm 0.75 \%$ of the final value, for the $5.5 \mu \mathrm{m}$ and $30.5 \mu \mathrm{m}$ thick AOBAE respectively. An interesting observation is that for a single layer sensor the capacitance value for $\mathrm{pH} 8.39$ is lower than for $\mathrm{pH} 7.12$, while the opposite is true for a double layer 
sensor, $\mathrm{pH} 8.39$ has a higher capacitance value then $\mathrm{pH} 7.12$. This behavior is in agreement with Figure 4, which display a simulation for the three different sensor cases in Figure 3.

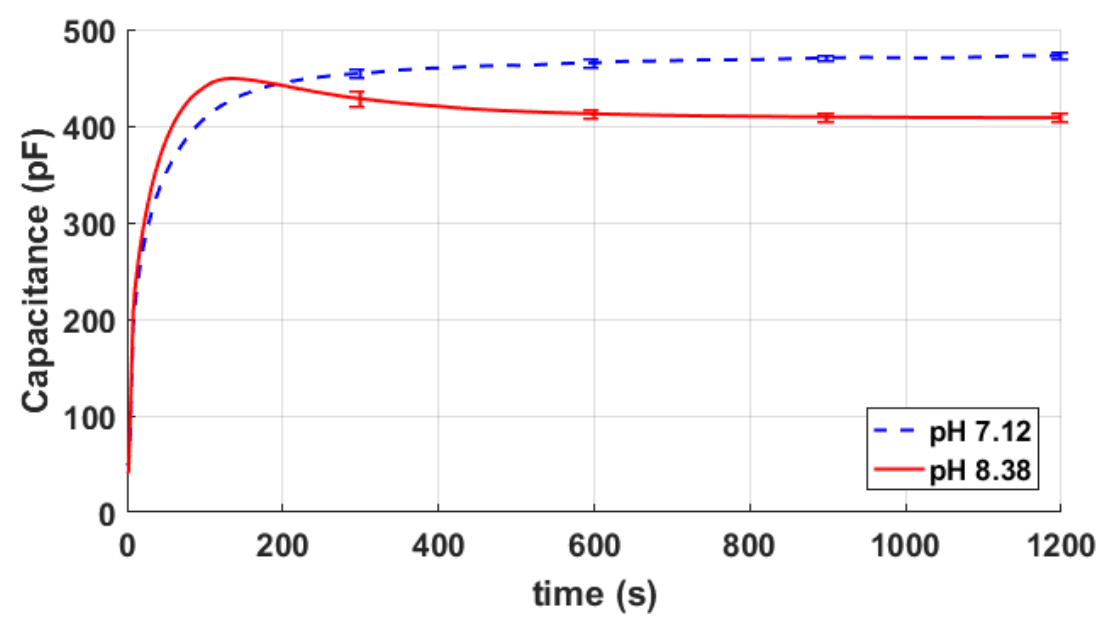

Figure 8. 10 measurements on a sensor with $18.5 \mu \mathrm{m}$ thick sensing layer, where the sensor was completely dried between the measurements. 5 measurements in each pH solution, $\mathrm{pH} 7.12$ and 8.38. Error bars are the standard deviation at 300,600, 900 and $1200 \mathrm{~s}$.

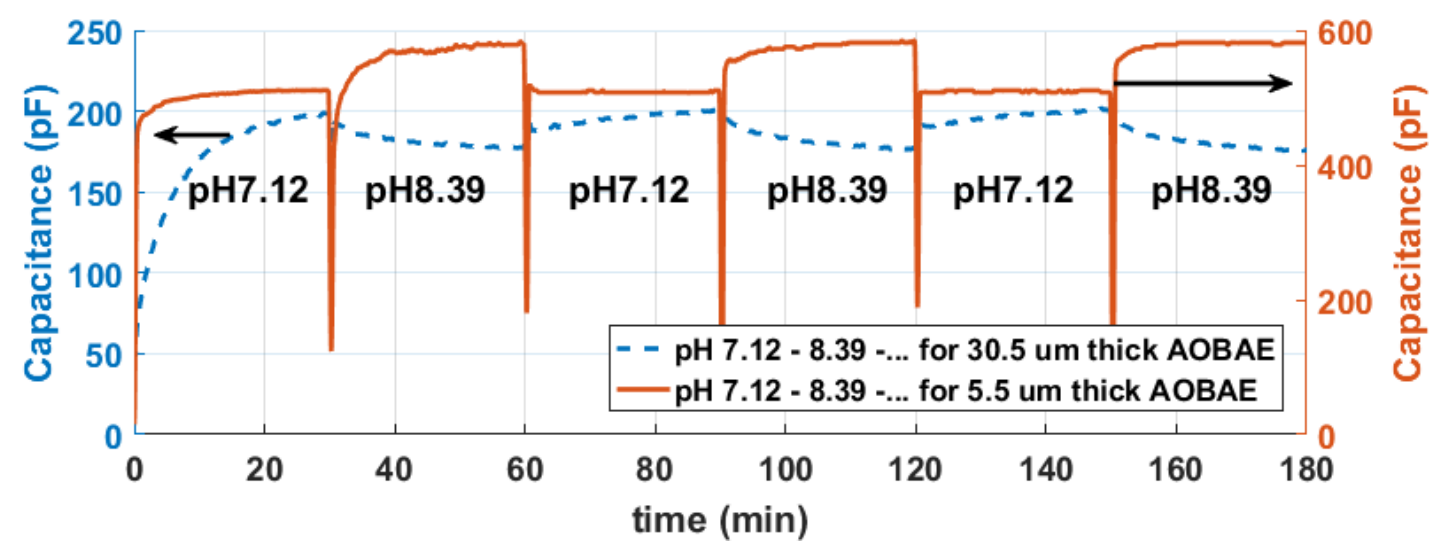

Figure 9. Cycling measurements of two sensors with AOBAE layer thicknesses of 5.5 and $30.5 \mu \mathrm{m}$ respectively. The $5.5 \mu \mathrm{m}$ sensor is a dual layer sensor and the $30.5 \mu \mathrm{m}$ sensor is a single layer sensor. For the single layer sensor the capacitance value for $\mathrm{pH} 8.39$ is lower than for $\mathrm{pH}$ 7.12. While for a double layer sensor it is the opposite. This is in agreement with the simulation (Figure 4). The dips in the graph are when the $\mathrm{pH}$ was changed.

Figure 10 shows a dual layer sensor with a $5.5 \mu \mathrm{m}$ thick sensing layer tested in $\mathrm{pH}$ between 2.94 and 11.80. As predicted by the simulation (Figure 4) the capacitance decreases as the AOBAE expands due to lower $\mathrm{pH}$. We also measured in $\mathrm{pH}$ 2.01, though the adhesion between the AOBAE and SU8 could not withstand the physical forces from swelling and the AOBAE layer started to delaminate. The sensor was allowed to dry slightly between changing of $\mathrm{pH}$ buffer solutions, which can be seen by the dips between each $\mathrm{pH}$. In the insets a and $\mathrm{b}$ in Figure 10, the behavior when changing $\mathrm{pH}$ is different. This comes from the fact that at position $b$ the sensor dried out more compared to position a, as indicated by the larger decrease in capacitance.

In Figure 11, the capacitance as a function of $\mathrm{pH}$ from Figure 10 is shown. The capacitance values are the last value for each $\mathrm{pH}$ before changing to a new $\mathrm{pH}$. There is an exponential relation between capacitance and the distance of measured material [25] as described in the Theory section, see Figure 2. Therefore, an exponential curve has been used for the curve fitting. However, since not all 
$\mathrm{pH}$ values in Figure 10 have stabilized, it should be seen more as a guideline for the sensor behavior. In addition, any changes to the sensors effective area $A$, spacing $G$ or the hydrogel thickness will alter this curve fitting.

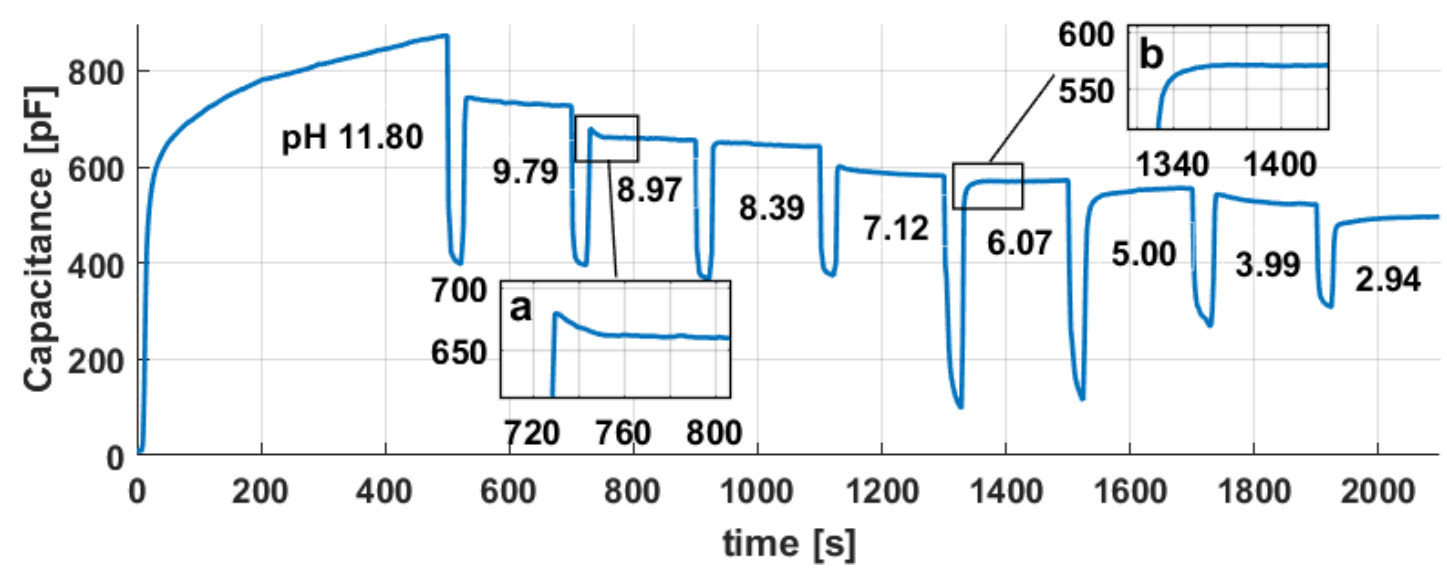

Figure 10. A $5.5 \mu \mathrm{m}$ thick dual layer sensor tested in $\mathrm{pH}$ from 11.80 to 2.94 . In the two insets a and $\mathrm{b}$ the behavior of the sensor when changing $\mathrm{pH}$ is different. This comes from that in $\mathrm{b}$ the sensor dried out more then in a, as can bee seen by the deeper dip. Which are from when the $\mathrm{pH}$ solutions are changed. The results are in agreement with the simulation for a dual layer sensor, see Figure 4 .

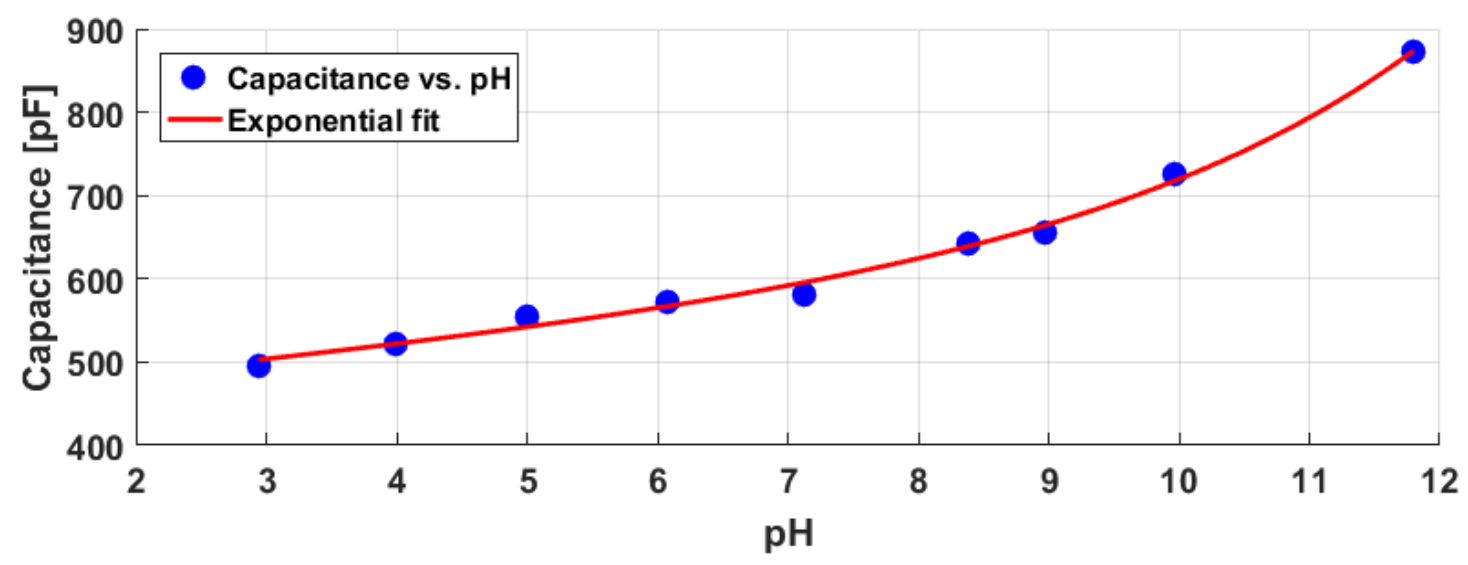

Figure 11. The graph show the capacitance value as a function of $\mathrm{pH}$ of the sensor measured in Figure 10. The points are the last capacitance value measured for each $\mathrm{pH}$ in Figure 11 before change of $\mathrm{pH}$ was made.

In Figure 12 a measurement of a $72 \mu \mathrm{m}$ thick AOBAE film in pH 6.07-8.89 is shown. The time constant for a hydrogel has a squared relation to the thickness [27] and the calculated time constant for the $72 \mu \mathrm{m}$ film is $>10 \mathrm{~h}$. Due to this long time constant, we chose to make the measurements for only $1 \mathrm{~h}$ for each $\mathrm{pH}$. Therefore it can be seen in the measurements that the $\mathrm{pH}$ values are not stable at the times when the $\mathrm{pH}$ are changed. Although the time of measurement per $\mathrm{pH}$ was short compared to the calculated time constant, the $\mathrm{pH}$ change is clearly seen in the inset of Figure 12. The results are in agreement of the simulation of a single layer sensor, that the capacitance should increase with lower $\mathrm{pH}$ values, see Figure 4. We also measured in $\mathrm{pH} 5.00$, although the AOBAE was observed to delaminate from the SU-8 layer. This happened at a higher $\mathrm{pH}$ then for the $5.5 \mu \mathrm{m}$ thick film, which started to delaminate at $\mathrm{pH}$ 2.01. The earlier delamination comes from that a thicker film exerts a larger physical force from swelling upon the substrate then a thinner. 


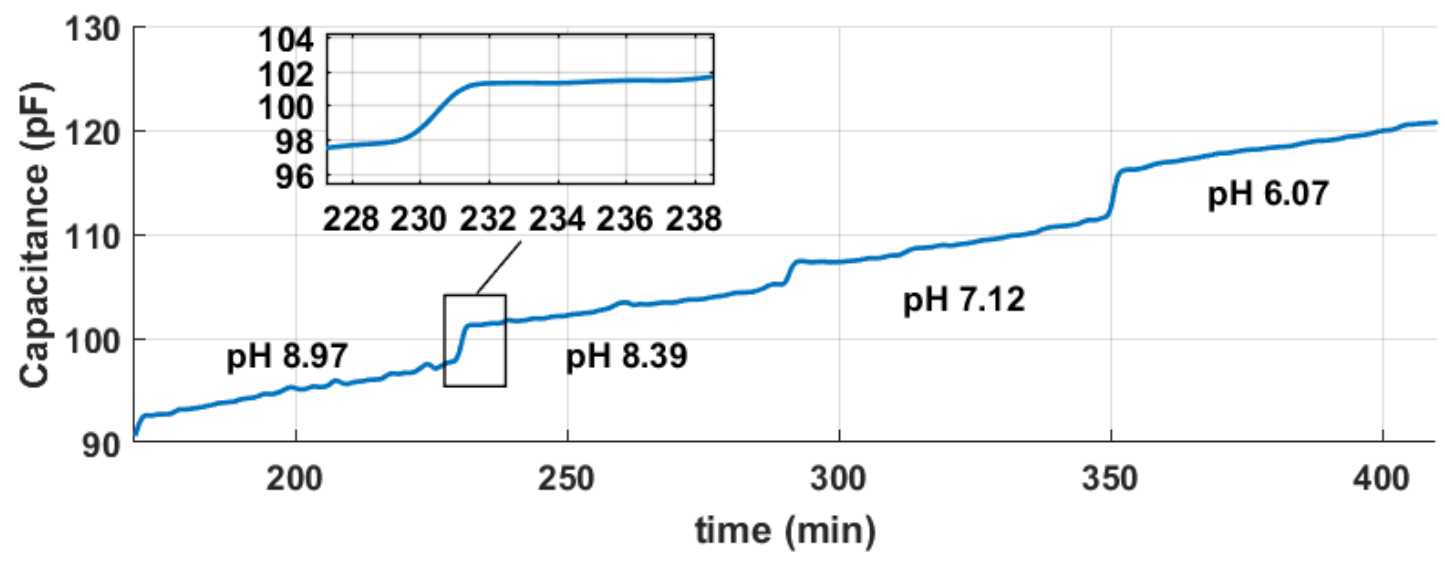

Figure 12. A $72 \mu \mathrm{m}$ thick single layer sensor tested in $\mathrm{pH}$ from 8.97 to 6.07. The sensor measurements are not stable at point of $\mathrm{pH}$ change. This because the time constant to equilibrium for this $72 \mu \mathrm{m}$ thick AOBAE film is very long, therefore we choose to only make $1 \mathrm{~h}$ measurement for each $\mathrm{pH}$. Though, the results are still in agreement with the simulation for a single layer sensor, see Figure 4.

\section{Discussion}

As stated earlier, the sensing mechanism is based on the permittivity change in AOBAE due to different uptake of water depending on the $\mathrm{pH}$ level. Because $\epsilon_{r}$ for water is $\sim 80$, compared to $\sim 5.5$ for the AOBAE, the capacitance change due to water uptake is very high, see Equation (1). For thicker layers, $E_{P D}<D+\Delta D$, the capacitance change comes only from the permittivity change of the hydrogel.

For thinner layers, $E_{P D}>D+\Delta D$, the effect is more complicated as part of the electric field penetrates into the water even for low $\mathrm{pH}$ levels. Then, as the hydrogel releases water at increasing $\mathrm{pH}$ level the effective permittivity is reduced in the hydrogel. At the same time the thickness of the hydrogel decreases and the electric field penetrates more into the water resulting in a permittivity increase. These two effects counteract each other and the resulting capacitance change can be anything from smaller to even reverse of what is expected. This is clearly displayed in Figure 9, where a $30.5 \mu \mathrm{m}$ thick hydrogel layer show the expected capacitance change but the $5.5 \mu \mathrm{m}$ layer shows the opposite.

To fabricate a sensor with the most predictable capacitance change, $E_{P D}$ should be enclosed in the hydrogel layer at all $\mathrm{pH}$ levels. Because, if $E_{P D}>D+\Delta D$ the sensor also measures upon the water which can be contaminated with other substances. These other substances may also enter the hydrogel if the particles are small enough to enter the network of the hydrogel, though it might be possible to build a filter ontop of the hydrogel to minimize this risk. The $E_{P D}$ is defined as $(W+G) / 3$ [34] and can be adjusted by changing the width, $W$, and gap, $G$, of the electrodes. Figure 4 shows the results from a simulation considering three cases; single layer, double layer or alternating, as described in the Theory section. In Figure 4, the capacitance of the single layer sensor (blue dots, solid line) is shown to linearly increase with decreasing $\mathrm{pH}$ levels (hydrogel absorbs water) as the effective permittivity increases. This case corresponds to measurements made on the $72 \mu \mathrm{m}$ hydrogel layer as seen in Figure 12. The simulated double layer case, Figure 4 (gray triangles, dashed line) show a reverse behaviour, hence a decreasing capacitance. This corresponds to the sensor with the $5.5 \mu \mathrm{m}$ layer in Figure 10. In the case of a sensor where the electric field is fully inside the hydrogel layer for some $\mathrm{pH}$ levels and partly in the water for others a mixed case will be the result. This means that one capacitance value cannot be used to determine $\mathrm{pH}$ levels with certainty as some high and low levels will result in the same capacitance value, see Figure 4 (diamonds).

We have chosen the electrode width and gap $(W$ and $G$ ) so that it was possible to build both single and double layer sensors from one interdigital pattern. This might not be optimal for a commercial sensor configuration. 
The response time of the sensor depends on the hydrogel thickness, $D$, as the swelling kinetics of a hydrogel is governed by a diffusion process [27]. This means that to design a fast sensor, a thin hydrogel layer is needed. This also means that the interdigital capacitance structure requires narrow electrodes width $(W)$ and gap $(G)$ in order to build a single layer sensor.

The response time can be examined in Figure 7. The first rapid, linear change in capacitance (see inset Figure 7) occurs when the sensor is inserted in the water and thereby instantly increasing the dielectric constant for the layer outside the AOBAE. This instant change is smallest for the $30.5 \mu \mathrm{m}$ layer because the electric field is enclosed more in the AOBAE relative to the thinner layers.

The response time can be further examined in Figures 9 and 10 where a small difference in capacitance is noted in the beginning after $\mathrm{pH}$ change, as marked in Figure 10. Here it should be noted that the change of $\mathrm{pH}$ buffer solution is not instantaneous but rather the sensor is removed from the beaker of one solution before immersed in the next. This short time makes the sensor start to sense air and dry out rather quickly. As can be seen in the insets of Figure 10, the starting capacitance level after a $\mathrm{pH}$ change is not the same as of the previous $\mathrm{pH}$ but slightly higher or even lower. This can be explained by the rapid drying out of the hydrogel, the longer time the sensor is in air the less water it retains and the lower the permittivity. The effect of air sensing and drying is not seen in Figure 12 where the $72 \mu \mathrm{m}$ thick AOBAE is thick enough to be outside the penetration depth $E_{P D}$ of the sensor.

A thinner sensor should be faster then a thicker one. Though, for Figure 10 the $5.5 \mu \mathrm{m}$ thick sensor it can be seen that $\mathrm{pH} 11.80$ has not yet stabilized during the first $500 \mathrm{~s}$. While, for the rest of the $\mathrm{pH}$ measurements the responses are faster. We believe that this is a physical effect that the less water a hydrogel contains the harder it has to de-swell. There can be several reasons why it is hard for a hydrogel to de-swell. The rate at which a change in hydration occurs can be limited by diffusive transport of protons or by mechanical relaxation of the polymer network as a result of water movement into or out of the gel [38]. The water content of the gel can be very low, thus, the flexibility of the network chains can be hindered. De-swelling from a more swollen to a less swollen state (here, e.g., when the outer $\mathrm{pH}$ is considerably increased) could be hindered, because the first outermost layer shrinks and becomes less permeable. Hence, this layer hinder the release of water from the deeper layers. Swelling from a less swollen or dry state is not hindered in this way, while first the external layers swells and lets the water enter deeper.

Repeatability tests were done to test if the sensors would be effected permanently due to drying out between measurements. A sensor was cycled 10 times between $\mathrm{pH} 7.12$ and 8.38 and was let to dry in between measurements, see Figure 8. The standard deviation for $\mathrm{pH} 7.12$ was $3.37 \mathrm{pF}$ and for $\mathrm{pH} 8.384 .69 \mathrm{pF}$, which is \pm 0.7 and $\pm 1.1 \%$ of the respectively stable capacitance value. The deviation of cycling without drying in-between, Figure 9 , is \pm 0.5 and $\pm 0.75 \%$ of the final value, for a 5.5 and $30.5 \mu \mathrm{m}$ thick AOBAE respectively. The conclusion is that that the sensor is stable enough to be used to repeatedly measure $\mathrm{pH}$ levels, and not just as a one-time disposable sensor.

A feature in Figure 8 is that one also can see a pronounced peak at $100 \mathrm{~s}$ for $\mathrm{pH} 8.38$ not seen for $\mathrm{pH}$ 7.12. We believe that this is a memory effect from when the sensor is submerged in $\mathrm{pH} 7.12$ and the AOBAE becomes more protonated (more expanded) and contains more anions from the buffer then when submerged in $\mathrm{pH}$ 8.38. Thus, when it dries out the sensor gets "stuck" with that protonation and anions concentration. Because it takes time for the AOBAE to soak up water (needed for the proton $\mathrm{H}^{+}$ to move freely). Therefore, the first capacitance response after it is again submerged in a higher $\mathrm{pH}$ will be as for $\mathrm{pH}$ 7.12. Then, as more water diffuses into the AOBAE the protons and anions can start to move freely and the sensor changes in response to the new $\mathrm{pH}$ value. This effect is not noticeable when the sensor goes from low towards high protonation levels. Or when the change of $\mathrm{pH}$ is so fast that the sensor does not have time to dry out and the $\mathrm{H}^{+}$protons can move freely all the time.

The AOBAE is shown to be responsive to a wide $\mathrm{pH}$ range, 2.94-11.80, see Figure 10. At a $\mathrm{pH}$ level of 2.01 the AOBAE started to delaminate from the SU8 layer as a result of the physical forces exerted due to the isotropic nature of swelling (i.e., the gel layer swells uniformly to same degree). 
However, there is a constraint in the lateral direction as it adheres to the substrate. It is possible that with a better adhesion between the layers, the sensor would function even at lower $\mathrm{pH}$ levels.

To summarize, single layer sensors are to be preferred, since they have the best sensitivity and most predictable outcome. Thinner hydrogel layer gives a faster response, although there is a risk that the sensor becomes a double layer sensor. A thick layer, $E_{P D}<<D+\Delta D$, have more resistance to physical damages, thus possible longer life time. Thick layers exerts a larger physical force on the substrate compared to thinner, but good adhesion is needed.

All steps for manufacturing of this sensor are compatible with roll-to-roll (R2R) processes. Hence, it is possible to mass-produce this sensor which may reduce the manufacturing cost.

\section{Conclusions}

We have shown that the sensor behavior varies with different electrical field penetration depths, $E_{P D}$, and hydrogel dry thickness, $D$, as well as maximum thickness change, $\Delta D$. Where we have concluded that depending on if $D+\Delta D$ is larger, smaller or about the same as $E_{P D}$, the sensor system will behave differently. The most predictable sensor system is built as a single layer sensor, i.e., $E_{P D}>D+\Delta D$. This has, to our knowledge, not been addressed in any earlier reports. An interdigital $\mathrm{pH}$ sensor that is suitable to be used in environmental monitoring systems has been built and characterized. The sensor utilizes a biocompatible hydrogel (AOBAE) as sensing material. This AOBAE swells or deswells (thus alters its relative permittivity) depending on the $\mathrm{pH}$ it is exposed to and is at least sensitive in the $\mathrm{pH}$ range 2.94-11.80.

Author Contributions: All authors conceived and designed the experiments. K.H. performed the experiments and analyzed the test data. M.S. provided advice on molecular design, curing conditions and interpretation of AOBAE response. K.H. wrote the manuscript, with the exception of the part about laser ablation, which was written by M.E.. H.A. and H.-E.N. provided advice on printing techniques and printed electronics. All authors have proofread the manuscript.

Acknowledgments: The Knowledge Foundation (KKS), the Interreg Sweden-Norway Program and Fiber Optic Valley are gratefully acknowledged for the financial support. Mats Sandberg's work was financed by The Swedish Foundation for Strategic Environmental Research (Mistra) in the research program Mistra TerraClean.

Conflicts of Interest: The authors declare no conflict of interest. The founding sponsors had no role in the design of the study, in the collection, analyses, or interpretation of data, in the writing of the manuscript and in the decision to publish the results.

\section{Abbreviations}

The following abbreviations are used in this manuscript:

$\begin{array}{ll}\text { AOBAE } & \text { Acryl-terminated oligo ( } \beta \text {-amino ester) } \\ \text { BDDA } & \text { Butanediol diacrylate } \\ \text { PIP } & \text { Piperazine } \\ \text { TPO } & \text { Diphenyl(2,4,6-trimethylbenzoyl)phosphine oxide } \\ \text { BRB } & \text { Britton-Robinson buffer }\end{array}$

\section{Appendix A. Simulation}

In the simulation the total sensor capacitance $C_{\text {tot }}$ is calculated as

$$
C_{\text {tot }}=C_{1}+C_{2} \begin{cases}C_{2}=0 & \text { if } D+\Delta D \geqslant E_{P D} \text { (single layer sensor) } \\ C_{2}=C_{2} & \text { if } D+\Delta D<E_{P D} \text { (double layer sensor) }\end{cases}
$$

where $C_{\text {tot }}$ is the total capacitance. $C_{1}$ is the capacitance contribution that comes from the hydrogel layer and $C_{2}$ is the capacitance contribution that comes from measure water (or air). Depending on the hydrogels start thickness, $D$. $C_{1}$ and $C_{2}$ will have different contribution within the penetration depth 
$E_{P D}$, where $E_{C 1 \%}$ is the contribution for $C_{1}$ and $E_{C 2 \%}$ for $C_{2}$ and $E_{C 1 \%}+E_{C 2 \%}=100 \%$ of the penetration depth $E_{P D}$. Taking this into account then Equation (A1) becomes

$$
C_{\text {tot }}=C_{1} E_{C 1 \%}+C_{2} E_{C 2 \%}
$$

As relative permittivity of the hydrogel we have used $\epsilon_{h}=5.5$, water $\epsilon_{W}=80$ and air $\epsilon_{\text {air }}=1$. And the effective relative permittivity $\epsilon_{h w}$ of water soaked hydrogel has been calculated with the linear formula

$$
\epsilon_{h w}=\frac{\left(\epsilon_{h}\left(100-\frac{s \%}{2}\right)+\epsilon_{w} \frac{s \%}{2}\right)}{100}
$$

where $s \%$ is the thickness increase in $\%$ of the hydrogel, $\epsilon_{h}\left(100-\frac{s \%}{2}\right)$ equals the permittivity part from the hydrogel and $\epsilon_{w} \frac{s_{\%}}{2}$ is the permittivity from the water uptake. The simulation is based on the capacitance equation $C=\epsilon_{r} \epsilon_{0} A / d$, where the physical dimension parameters $\mathrm{A}, \mathrm{d}$ and $\epsilon_{0}$ are constant therefore we can set $\epsilon_{0} A / d=k$, the capacitance $C$ then becomes $C=\epsilon_{r} k$. Thus for this simulation we can substitute $C_{1}$ in formula (A2) with formula (A3) and $C_{2}$ with $\epsilon_{w} \frac{s \%}{2}$ since $C_{2}$ is water (or air if completely dry). But then we also needs to adjust the start thicknesses $E_{\mathrm{C} 1 \%}$ and $E_{\mathrm{C} 2 \%}$, with the swelling degree of the hydrogel. $C_{t o t}$ then becomes

$$
\begin{gathered}
C_{t o t}=C_{1} E_{C 1 \%}+C_{2} E_{C 2 \%}= \\
\frac{\left(\epsilon_{h}\left(100-\frac{S \%}{2}\right)+\epsilon_{w} \frac{S_{\%}}{2}\right)}{100} k\left(E_{C 1 \%}+E_{C 2 \%} \frac{S \%}{100}\right)+\epsilon_{w} \frac{S \%}{2} k\left(E_{C 2 \%}-E_{C 1 \%} \frac{S \%}{100}\right) \\
\begin{cases}\epsilon_{w} \frac{S \%}{2}=0 & \text { if } D+\Delta D \geqslant E_{P D} \text { (single layer sensor) } \\
\epsilon_{w} \frac{S \%}{2}=\epsilon_{w} \frac{S \%}{2} & \text { if } D+\Delta D<E_{P D} \text { (double layer sensor) } \\
\epsilon_{w} \frac{S \%}{2}=\epsilon_{a i r}=1 & \text { if } s \%=0\end{cases}
\end{gathered}
$$

\section{References}

1. Dimpe, K.M.; Nomngongo, P.N. Current sample preparation methodologies for analysis of emerging pollutants in different environmental matrices. TrAC Trends Anal. Chem. 2016, 82, 199-207. [CrossRef]

2. Dehua, W.; Pan, L.; Bo, L.; Zeng, G. Water quality automatic monitoring system based on GPRS data communications. Procedia Eng. 2012, 28, 840-843. [CrossRef]

3. Luo, X.; Yang, J.; Chai, L. Water pollution source detection in wireless sensor networks. In Proceedings of the 2015 IEEE International Conference on Information and Automation, Lijiang, China, 8-10 August 2015; pp. 2311-2315.10.1109/ICInfA.2015.7279671. [CrossRef]

4. Yang, X.; Ong, K.G.; Dreschel, W.R.; Zeng, K.; Mungle, C.S.; Grimes, C.A. Design of a wireless sensor network for long-term, in-situ monitoring of an aqueous environment. Sensors 2002, 2, 455-472. [CrossRef]

5. Lukman, S.; Essa, M.; Mu'azu, N.D.; Bukhari, A.; Basheer, C. Adsorption and desorption of heavy metals onto natural clay material: influence of initial pH. J. Environ. Sci. Technol. 2013, 6, 1-5. [CrossRef]

6. Balintova, M.; Petrilakova, A.; Singovszka, E. Study of metals distribution between water and sediment in the Smolnik Creek (Slovakia) contaminated by acid mine drainage. Chem. Eng. Trans. 2012, 28, 73-78.

7. Pastor, J.; Hernández, A.J. Heavy metals, salts and organic residues in old solid urban waste landfills and surface waters in their discharge areas: Determinants for restoring their impact. J. Environ. Manag. 2012, 95, S42-S49. [CrossRef] [PubMed]

8. Wu, Q.; Leung, J.Y.; Geng, X.; Chen, S.; Huang, X.; Li, H.; Huang, Z.; Zhu, L.; Chen, J.; Lu, Y. Heavy metal contamination of soil and water in the vicinity of an abandoned e-waste recycling site: Implications for dissemination of heavy metals. Sci. Total Environ. 2015, 506, 217-225. [CrossRef] [PubMed]

9. Zheng, J.; Chen, K.h.; Yan, X.; Chen, S.J.; Hu, G.C.; Peng, X.W.; Yuan, J.G.; Mai, B.X.; Yang, Z.Y. Heavy metals in food, house dust, and water from an e-waste recycling area in South China and the potential risk to human health. Ecotoxicol. Environ. Saf. 2013, 96, 205-212. [CrossRef] [PubMed]

10. Connell, D.W.; Miller, G.J. Chemistry and Ecotoxicology of Pollution; John Wiley \& Sons: Hoboken, NJ, USA, 1984; Volume 65. 
11. Khan, M.I.; Mukherjee, K.; Shoukat, R.; Dong, H. A review on pH sensitive materials for sensors and detection methods. Microsyst. Technol. 2017, 23, 4391-4404. [CrossRef]

12. Huang, W.D.; Cao, H.; Deb, S.; Chiao, M.; Chiao, J.C. A flexible pH sensor based on the iridium oxide sensing film. Sens. Actuators A Phys. 2011, 169,1-11. [CrossRef]

13. Kaempgen, M.; Roth, S. Transparent and flexible carbon nanotube/polyaniline pH sensors. J. Electroanal. Chem. 2006, 586, 72-76. [CrossRef]

14. Gerlach, G.; Guenther, M.; Sorber, J.; Suchaneck, G.; Arndt, K.F.; Richter, A. Chemical and pH sensors based on the swelling behavior of hydrogels. Sens. Actuators B Chem. 2005, 111, 555-561. [CrossRef]

15. Hammarling, K.; Sandberg, M.; Engholm, M.; Andersson, H.; Nilsson, H.E. Synthesis, Curing Behavior and Swell Tests of $\mathrm{pH}$-Responsive Coatings from Acryl-Terminated Oligo ( $\beta$-Amino Esters). Chemosensors 2018, 6, 10. [CrossRef]

16. Mamishev, A.V.; Sundara-Rajan, K.; Yang, F.; Du, Y.; Zahn, M. Interdigital sensors and transducers. Proc. IEEE 2004, 92, 808-845. [CrossRef]

17. Ong, J.B.; You, Z.; Mills-Beale, J.; Tan, E.L.; Pereles, B.D.; Ong, K.G. A wireless, passive embedded sensor for real-time monitoring of water content in civil engineering materials. IEEE Sens. J. 2008, 8, 2053-2058. [CrossRef]

18. Kim, J.S.; others. Analysis of dielectric sensors for the cure monitoring of resin matrix composite materials. Sens. Actuators B Chem. 1996, 30, 159-164.

19. Ong, K.G.; Grimes, C.; Robbins, C.; Singh, R. Design and application of a wireless, passive, resonant-circuit environmental monitoring sensor. Sens. Actuators A Phys. 2001, 93, 33-43. [CrossRef]

20. Steindl, R.; Pohl, A.; Seifert, F. Impedance loaded SAW sensors offer a wide range of measurement opportunities. IEEE Trans. Microw. Theory Tech. 1999, 47, 2625-2629. [CrossRef]

21. Igreja, R.; Dias, C. Analytical evaluation of the interdigital electrodes capacitance for a multi-layered structure. Sens. Actuators A Phys. 2004, 112, 291-301. [CrossRef]

22. Von Guggenberg, P.; Zaretsky, M. Estimation of one-dimensional complex-permittivity profiles: A feasibility study. J. Electrostat. 1995, 34, 263-277. [CrossRef]

23. Ngo, T.T.; Bourjilat, A.; Claudel, J.; Kourtiche, D.; Nadi, M. Design and realization of a planar interdigital microsensor for biological medium characterization. In Next Generation Sensors and Systems; Springer: Basel, Switzerland, 2016; pp. 23-54.

24. Starzyk, F. Parametrisation of interdigit comb capacitor for dielectric impedance spectroscopy. Arch. Mater. Sci. Eng. 2008, 34, 31-34.

25. Li, X.B.; Larson, S.D.; Zyuzin, A.S.; Mamishev, A.V. Design principles for multichannel fringing electric field sensors. IEEE Sens. J. 2006, 6, 434-440. [CrossRef]

26. Puers, R. Capacitive sensors: When and how to use them. Sens. Actuators A Phys. 1993, 37, 93-105. [CrossRef]

27. Richter, A.; Paschew, G.; Klatt, S.; Lienig, J.; Arndt, K.F.; Adler, H.J.P. Review on hydrogel-based pH sensors and microsensors. Sensors 2008, 8, 561-581. [CrossRef] [PubMed]

28. Deligkaris, K.; Tadele, T.S.; Olthuis, W.; van den Berg, A. Hydrogel-based devices for biomedical applications. Sens. Actuators B Chem. 2010, 147, 765-774. [CrossRef]

29. Tokarev, I.; Minko, S. Stimuli-responsive hydrogel thin films. Soft Matter 2009, 5, 511-524. [CrossRef]

30. Richter, A. Hydrogels for actuators. In Hydrogel Sensors and Actuators; Springer Science \& Business Media: Berlin, Germany, 2009; pp. 221-248.

31. Banna, M.H.; Imran, S.; Francisque, A.; Najjaran, H.; Sadiq, R.; Rodriguez, M.; Hoorfar, M. Online drinking water quality monitoring: Review on available and emerging technologies. Crit. Rev. Environ. Sci. Technol. 2014, 44, 1370-1421. [CrossRef]

32. Rahman, M.S.A.; Mukhopadhyay, S.C.; Yu, P.L. Novel planar interdigital sensors. In Novel Sensors for Food Inspection: Modelling, Fabrication and Experimentation; Springer: Berlin, Germany, 2014; pp. 11-35.

33. Mamishev, A.V.; Lesieutre, B.C.; Zahn, M. Optimization of multi-wavelength interdigital dielectrometry instrumentation and algorithms. IEEE Trans. Dielectr. Electr. Insul. 1998, 5, 408-420. [CrossRef]

34. Mamishev, A.V.; Du, Y.; Bau, J.H.; Lesieutre, B.C.; Zahn, M. Evaluation of diffusion-driven material property profiles using three-wavelength interdigital sensor. IEEE Trans. Dielectr. Electr. Insul. 2001, 8, 785-798. [CrossRef]

35. Britton, H.T.S.; Robinson, R.A. CXCVIII.-Universal buffer solutions and the dissociation constant of veronal. J. Chem. Soc. (Resumed) 1931, pp. 1456-1462. [CrossRef] 
36. Dorf, R.C. Electronics, Power Electronics, Optoelectronics, Microwaves, Electromagnetics, and Radar; CRC Press: Boca Raton, FL, USA, 2006.

37. Tanaka, T.; Fillmore, D.J. Kinetics of swelling of gels. J. Chem. Phys. 1979, 70, 1214-1218. [CrossRef]

38. Lesho, M.J.; Sheppard, N.F., Jr. A method for studying swelling kinetics based on measurement of electrical conductivity. Polym. Gels Netw. 1998, 5, 503-523. [CrossRef]

(C) 2018 by the authors. Licensee MDPI, Basel, Switzerland. This article is an open access article distributed under the terms and conditions of the Creative Commons Attribution (CC BY) license (http:/ / creativecommons.org/licenses/by/4.0/). 